\title{
Cyclobis(paraquat-p-phenylene)-Based [2]Catenanes Prepared by Kinetically Controlled Reactions Involving Alkynes
}

Ognjen Š. Miljanić, William R. Dichtel, Shahab Mortezaei, and J. Fraser Stoddart*

California NanoSystems Institute and Department of Chemistry and Biochemistry, University of California, Los Angeles, 405 Hilgard Avenue, Los Angeles, CA 90095

\section{Supporting Information REVISED VERSION}

\begin{tabular}{|c|}
\hline Correspondence Address \\
\hline Professor J Fraser Stoddart \\
California NanoSystems Institute and \\
Department of Chemistry and Biochemistry \\
University of California, Los Angeles \\
405 Hilgard Avenue \\
Los Angeles, CA 90095-1569 (USA) \\
Tel: (+1)-310-206-7078 \\
Fax: (+1)-310-206-1843 \\
Email: $\underline{\text { stoddart@ chem.ucla.edu }}$ \\
\hline
\end{tabular}


General Methods. All reagents were purchased from commercial suppliers (Aldrich or Fisher) and used without further purification. Cyclobis(paraquat-p-phenylene) hexafluorophosphate, ${ }^{1} \quad$ 1,5-bis[2-(2-(2-hydroxyethoxy)ethoxy)ethoxy]naphthalene, ${ }^{2}$ and 1,1'-[1,4-phenylenebis(methylene)]bis-4,4'-bipyridinium hexafluorophosphate ${ }^{3}$ were prepared according to literature procedures. Microwave-assisted reactions were performed in a CEM Discover System 908005, producing monochromatic microwave radiation with the frequency of $2455 \mathrm{MHz}$. Thin layer chromatography (TLC) was performed on silica gel $60 \mathrm{~F}_{254}$ (E. Merck). Column chromatography was performed on silica gel 60F (Merck 9385, 0.040-0.063 mm). Melting points were recorded on an Electrothermal 9100 instrument in open capillary tubes and are uncorrected. Nuclear magnetic resonance (NMR) spectra were recorded at $25{ }^{\circ} \mathrm{C}$ on a Bruker Avance 400 and 500 spectrometers, with working frequencies of 400 and $500 \mathrm{MHz}$ for ${ }^{1} \mathrm{H}$, and 100 and $125 \mathrm{MHz}$ for ${ }^{13} \mathrm{C}$ nuclei respectively. Chemical shifts are reported in $\mathrm{ppm}$ relative to the signals corresponding to the residual non-deuterated solvents $\left(\mathrm{CDCl}_{3}: \delta 7.26 \mathrm{ppm}, \mathrm{CD}_{3} \mathrm{CN}: \delta 1.93\right.$ ppm). All ${ }^{13} \mathrm{C}$ spectra were recorded with the simultaneous decoupling of proton nuclei. Fast atom bombardment mass spectra were obtained on a JEOL JMS-600H high resolution mass spectrometer equipped with a FAB probe.

Scheme S1. Synthesis of DNP Dialkyne Derivative 2.

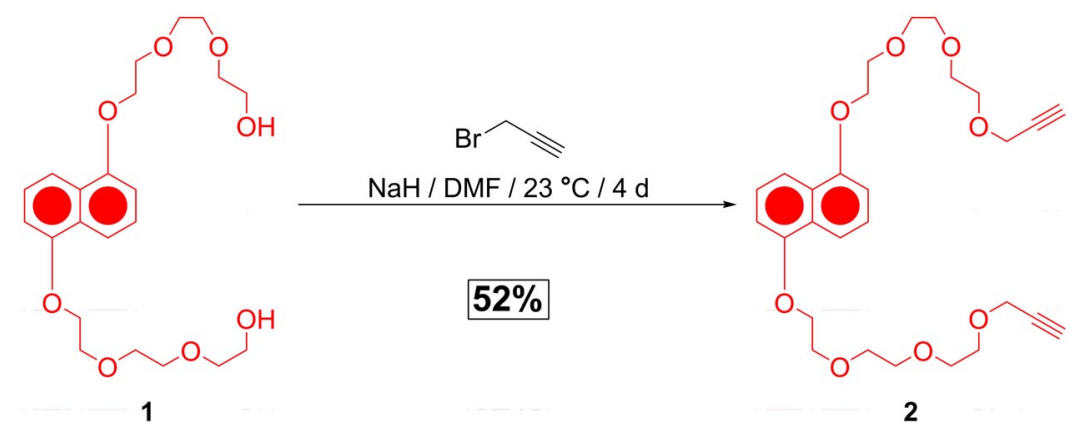

Preparation of 1,5-Bis(2-(2-(2-(prop-2-ynyloxy)ethoxy)ethoxy)ethoxy)naphthalene 2:

Solid $\mathrm{NaH}$ (362 mg, $15.1 \mathrm{mmol}$ ) was added to the solution of 1,5-bis[2-(2-(2hydroxyethoxy)ethoxy)ethoxy]naphthalene ${ }^{2}(\mathbf{1})(1.60 \mathrm{~g}, 3.78 \mathrm{mmol})$ in dry DMF (50 mL). The resulting suspension was stirred at $23{ }^{\circ} \mathrm{C}$ until the evolution of gas ceased (approx. 25 
min). A solution of propargyl bromide in xylene ( $80 \%$ by weight, $4.20 \mathrm{~mL}, 37.8 \mathrm{mmol}$ ) was injected using a syringe. The mixture was stirred at $23{ }^{\circ} \mathrm{C}$ for $4 \mathrm{~d}$, during which time it gradually turned brown. The reaction was stopped by the addition of $\mathrm{MeOH}$, and the solvents were removed in vacuo. Crude brown oil was first filtered through a plug of silica, eluting with $\mathrm{Me}_{2} \mathrm{CO}$, and then subjected to chromatography on silica (hexane/EtOAc = $50 / 50$ ) to provide 2 as a brown oil $(0.98 \mathrm{~g}, 52 \%)$. 2: $\mathrm{MS}(\mathrm{FAB}+): \mathrm{m} / \mathrm{z}$ (rel intensity) $=502$ (16\%), 501 (48), $500\left(\mathrm{M}^{+}, 100\right), 460$ (4), 433 (16), 419 (18). ${ }^{1} \mathrm{H}$ NMR (500 MHz, $\mathrm{CDCl}_{3}$ ): $\delta 7.86\left(\mathrm{~d},{ }^{3} J(\mathrm{H}, \mathrm{H})=8.9 \mathrm{~Hz}, 2 \mathrm{H}, \mathrm{DNP}\right.$ aryl $\left.-\mathrm{H} p-\mathrm{O}\right), 7.34\left(\mathrm{t},{ }^{3} J(\mathrm{H}, \mathrm{H})=8.2 \mathrm{~Hz}, 2 \mathrm{H}, \mathrm{DNP}\right.$ aryl $-\mathrm{H} m-\mathrm{O}), 6.84\left(\mathrm{~d},{ }^{3} J(\mathrm{H}, \mathrm{H})=7.6 \mathrm{~Hz}, 2 \mathrm{H}\right.$, DNP aryl $\left.-\mathrm{H} o-\mathrm{O}\right), 4.29\left(\mathrm{t},{ }^{3} J(\mathrm{H}, \mathrm{H})=4.9\right.$ $\left.\mathrm{Hz}, 4 \mathrm{H}, \mathrm{DNP}-\mathrm{OCH} H_{2}\right), 4.19\left(\mathrm{~d},{ }^{4} J(\mathrm{H}, \mathrm{H})=2.4 \mathrm{~Hz}, 4 \mathrm{H}\right.$, propargyl $\left.\mathrm{CH}_{2}\right), 3.99\left(\mathrm{t},{ }^{3} J(\mathrm{H}, \mathrm{H})=\right.$ $4.9 \mathrm{~Hz}, 4 \mathrm{H}), 3.81-3.79(\mathrm{~m}, 4 \mathrm{H}), 3.71-3.68(\mathrm{~m}, 8 \mathrm{H}), 2.41\left(\mathrm{t},{ }^{4} \mathrm{~J}(\mathrm{H}, \mathrm{H})=2.4 \mathrm{~Hz}, 2 \mathrm{H}, \equiv \mathrm{C}-H\right)$. ${ }^{13} \mathrm{C}$ NMR $\left(125 \mathrm{MHz}, \mathrm{CDCl}_{3}\right): \delta 154.23,126.67,124.96,114.51,105.56,79.56(C \equiv \mathrm{C}-\mathrm{H})$, $74.39(\mathrm{C} \equiv C-\mathrm{H}), 70.88,70.63,70.37,69.73,69.02,67.81,58.29$. HRMS Calcd for $\mathrm{C}_{28} \mathrm{H}_{36} \mathrm{O}_{8}: 500.2410$. Found: 500.2425.

Scheme S2. Microwave-Assisted Synthesis of Catenane $3 \cdot 4 \mathrm{PF}_{6}$.

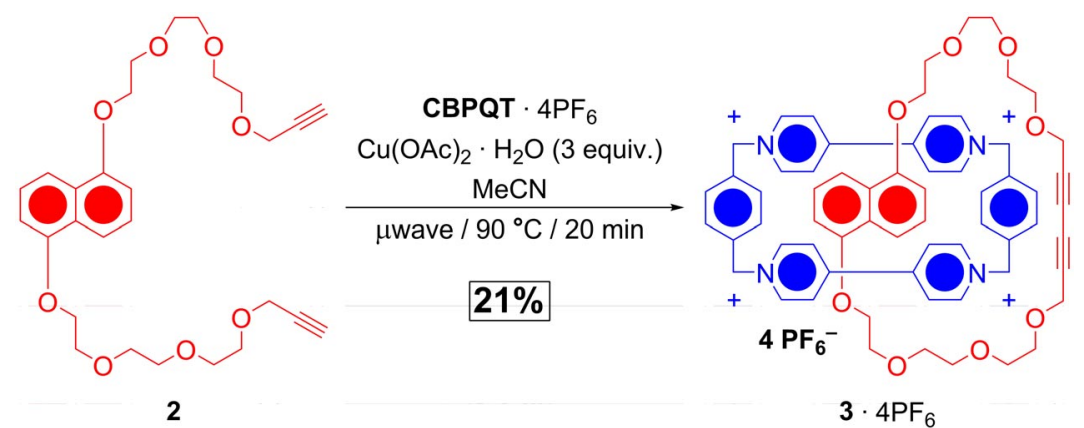

Microwave-Assisted Preparation of the [2]Catenane 3 $4 \mathrm{PFF}_{6}$ :

The DNP derivative 2 (15.0 mg, $0.03 \mathrm{mmol})$, CBPQT · 4PF 6 (10.0 mg, $0.009 \mathrm{mmol})$, and $\mathrm{Cu}(\mathrm{OAc})_{2} \cdot \mathrm{H}_{2} \mathrm{O}(8.0 \mathrm{mg}, 0.04 \mathrm{mmol})$ were dissolved in $\mathrm{MeCN}(3.0 \mathrm{~mL})$, forming a deep greenish-blue solution. The mixture was transferred into a $5 \mathrm{~mL}$ Intellivent thick-walled vial, sealed, and exposed to microwave irradiation for $20 \mathrm{~min}$ at $90{ }^{\circ} \mathrm{C}$. After this time, a significant amount of black precipitate was observed in the reaction vial and the color of the solution changed to purple. The crude reaction mixture was filtered through silica, 
eluting with a $1 \%$ solution of $\mathrm{NH}_{4} \mathrm{PF}_{6}$ in $\mathrm{Me}_{2} \mathrm{CO}$. The eluent was then treated with cold $\mathrm{H}_{2} \mathrm{O}$ to precipitate a purple solid. This solid was purified by column chromatography, eluting first with EtOAc (to remove the uncharged species), then continuing with $\mathrm{Me}_{2} \mathrm{CO}$, and ending with $2 \%$ solution of $\mathrm{NH}_{4} \mathrm{PF}_{6}$ in $\mathrm{Me}_{2} \mathrm{CO}$. The purple band was collected and was again treated with cold $\mathrm{H}_{2} \mathrm{O}$, precipitating a purple solid. After air-drying, this material was identified as the [2] catenane $3 \cdot 4 \mathrm{PF}_{6}\left(3.1 \mathrm{mg}, 21 \%, \mathrm{mp} 185{ }^{\circ} \mathrm{C}\right.$ with decomp.). Performing the same reaction at $23{ }^{\circ} \mathrm{C}$ in a round-bottom flask for $4 \mathrm{~d}$ provided [2] catenane $3 \cdot 4 \mathrm{PF}_{6}$ in $14 \%$ yield. Spectroscopic data collected for the two samples were identical. 3 - 4PF $: \mathrm{MS}(\mathrm{FAB}+): m / z$ (rel intensity) $=1453\left(\left[3 \cdot 3 \mathrm{PF}_{6}\right], 41 \%\right), 1308([3$. 2PF 6 , 68), $1163\left(\left[3 \cdot \mathrm{PF}_{6}\right], 24\right), 955\left(\left[\mathbf{C B P Q T} \cdot 3 \mathrm{PF}_{6}\right], 18\right), 810\left(\left[\mathbf{C B P Q T} \cdot 2 \mathrm{PF}_{6}\right], 100\right)$, $665\left(\left[\mathbf{C B P Q T} \cdot \mathrm{PF}_{6}\right], 58\right) .{ }^{1} \mathrm{H}$ NMR $\left(500 \mathrm{MHz}, \mathrm{CD}_{3} \mathrm{CN}\right): \delta 9.07$ (br s, 4H, $\alpha-\mathrm{CBPQT}^{4+}-$

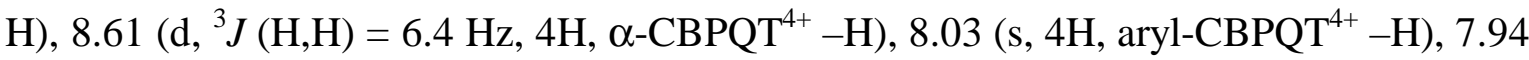
$\left(\mathrm{s}, 4 \mathrm{H}\right.$, aryl-CBPQT $\left.{ }^{4+}-\mathrm{H}\right), 7.42\left(\mathrm{br} \mathrm{d},{ }^{3} J(\mathrm{H}, \mathrm{H})=5.0 \mathrm{~Hz}, 4 \mathrm{H}, \beta-\mathrm{CBPQT}^{4+}-\mathrm{H}\right), 7.23(\mathrm{br} \mathrm{d}$, $\left.{ }^{3} J(\mathrm{H}, \mathrm{H})=5.0 \mathrm{~Hz}, 4 \mathrm{H}, \beta-\mathrm{CBPQT}^{4+}-\mathrm{H}\right), 6.27\left(\mathrm{~d},{ }^{3} J(\mathrm{H}, \mathrm{H})=7.6 \mathrm{~Hz}, 2 \mathrm{H}, \mathrm{DNP}\right.$ aryl $-\mathrm{H} o-$ O), $5.99\left(\mathrm{t},{ }^{3} J(\mathrm{H}, \mathrm{H})=7.9 \mathrm{~Hz}, 2 \mathrm{H}, \mathrm{DNP}\right.$ aryl $\left.-\mathrm{H} m-\mathrm{O}\right), 5.71\left(\mathrm{~s}, 8 \mathrm{H}, \mathrm{CBPQT}^{4+}\right.$ benzyl H$)$, $5.70\left(\mathrm{~s}, 8 \mathrm{H}, \mathrm{CBPQT}^{4+}\right.$ benzyl H), 4.31 (br s, 4H), 4.22 (br s, $\left.4 \mathrm{H}\right), 4.02\left(\mathrm{t},{ }^{3} J(\mathrm{H}, \mathrm{H})=4.0\right.$ Hz, 4H), 3.85 (br s, 4H), 3.69 (s, 4H, propargyl $\mathrm{CH}_{2}$ ), 3.62 (br s, 4H), 3.29 (br s, 4H), 2.42 $\left(\mathrm{d},{ }^{3} J(\mathrm{H}, \mathrm{H})=8.2 \mathrm{~Hz}, 2 \mathrm{H}, \mathrm{DNP}\right.$ aryl $\left.-\mathrm{H} p-\mathrm{O}\right)$. HRMS Calcd for $\mathrm{C}_{64} \mathrm{H}_{66} \mathrm{~N}_{4} \mathrm{O}_{8} \mathrm{P}_{3} \mathrm{~F}_{18}([3$. $\left.\left.3 \mathrm{PF}_{6}\right]\right):$ 1453.381. Found: 1453.385 .

Scheme S3. Synthesis of Catenane $3 \cdot 4 \mathrm{PF}_{6}$ by Tandem Hetero-Catenation.
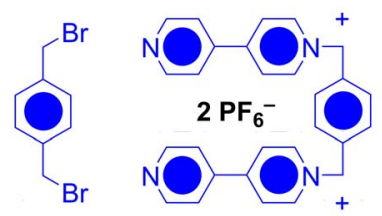

$5 \cdot 2 \mathrm{PF}_{6}$

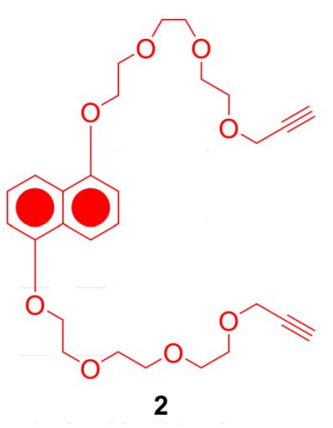

$\frac{\mathrm{Cu}(\mathrm{OAc})_{2} \cdot \mathrm{H}_{2} \mathrm{O} \text { (3 equiv.) }}{\mathrm{DMF} / 23^{\circ} \mathrm{C} / 5 \mathrm{~d}}$

2) $\mathrm{NH}_{4} \mathrm{PF}_{6} / \mathrm{H}_{2} \mathrm{O}$

$5 \%$

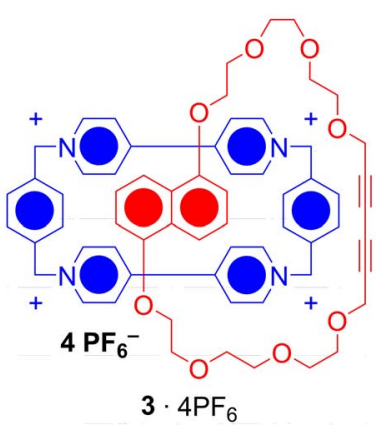

$3 \cdot 4 \mathrm{PF}_{6}$

Preparation of the [2]Catenane $3 \cdot 4 \mathrm{PF}_{6}$ by Tandem Hetero-Catenation:

The DNP derivative 2 (150 mg, $0.3 \mathrm{mmol}$ ), 1,4-bisbromomethylbenzene 4 (26.4 mg, 0.1 mmol), 1,1'-[1,4-phenylenebis(methylene)]bis-4,4'-bipyridinium hexafluorophosphate 5 . 
$2 \mathrm{PF}_{6}(70.6 \mathrm{mg}, 0.1 \mathrm{mmol})$, and $\mathrm{Cu}(\mathrm{OAc})_{2} \cdot \mathrm{H}_{2} \mathrm{O}(180 \mathrm{mg}, 0.9 \mathrm{mmol})$ were combined in DMF $(5.0 \mathrm{~mL})$. The mixture was stirred at $23{ }^{\circ} \mathrm{C}$ for $5 \mathrm{~d}$. During the course of reaction, the solution gradually changed color from brownish-yellow to purple, and precipitation was observed. After the completion of the reaction, the crude reaction mixture was filtered through a plug of silica ( $1 \%$ solution of $\mathrm{NH}_{4} \mathrm{PF}_{6}$ in $\left.\mathrm{Me}_{2} \mathrm{CO}\right)$ to provide a reddish-purple solution. The crude catenane was precipitated by the addition of $\mathrm{H}_{2} \mathrm{O}$. This chromatography-precipitation-filtration sequence was repeated two more times, to provide ultimately the [2] catenane $3 \cdot 4 \mathrm{PF}_{6}$ as a purple solid $(8.0 \mathrm{mg}, 5 \%)$. Spectroscopic data for this material were identical to those obtained for the product isolated from the microwaveassisted reaction.

Scheme S4. Synthesis of DNP Azido Derivative S1.

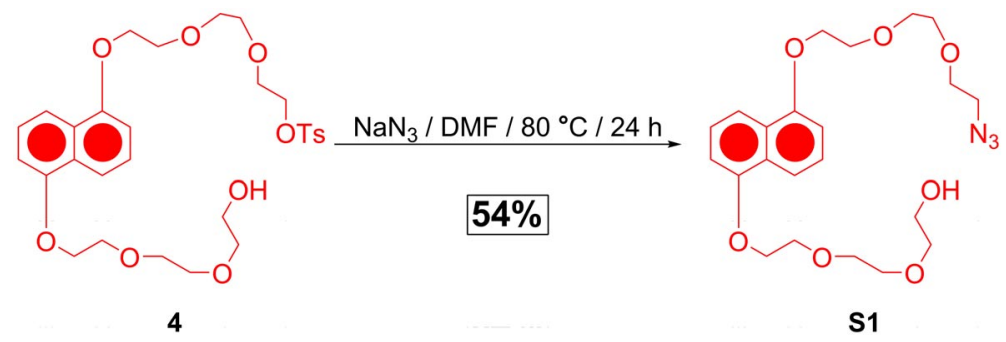

\section{Preparation of 1-(2-(2-(2-azidoethoxy)ethoxy)ethoxy)-5-(2-(2-(2-hydroxyethoxy)- ethoxy)ethoxy)naphthalene S1:}

A solution of tosylate $4^{4}(120 \mathrm{mg}, 0.207 \mathrm{mmol})$, and $\mathrm{NaN}_{3}(27.0 \mathrm{mg}, 0.415 \mathrm{mmol})$ in dry DMF (15 mL) was heated at $80{ }^{\circ} \mathrm{C}$ for $2 \mathrm{~d}$. After cooling, the solution was diluted with $\mathrm{H}_{2} \mathrm{O}(50 \mathrm{~mL})$ and EtOAc $(50 \mathrm{~mL})$ was added. The layers were separated and the organic solution was washed with two portions of a saturated aqueous solution of $\mathrm{NH}_{4} \mathrm{Cl}$, followed by drying $\left(\mathrm{MgSO}_{4}\right)$. The crude product, obtained after the removal of the solvent, was subjected to chromatography on silica (EtOAc) to provide $51.0 \mathrm{mg}$ of $\mathbf{S 1}$ as a viscous orangish oil (55\%). S1: MS (FAB+): $m / z$ (rel intensity) = $449\left(\mathbf{M}^{+}, 100 \%\right), 422(36), 317$ (11). ${ }^{1} \mathrm{H}$ NMR $\left(500 \mathrm{MHz}, \mathrm{CDCl}_{3}\right): \delta 7.82\left(\mathrm{~d},{ }^{3} J(\mathrm{H}, \mathrm{H})=8.2 \mathrm{~Hz}, 1 \mathrm{H}, \mathrm{DNP}\right.$ aryl $\left.-\mathrm{H} p-\mathrm{O}\right)$, $7.81\left(\mathrm{~d},{ }^{3} J(\mathrm{H}, \mathrm{H})=8.2 \mathrm{~Hz}, 1 \mathrm{H}, \mathrm{DNP}\right.$ aryl $\left.-\mathrm{H} p-\mathrm{O}\right), 7.30\left(\mathrm{t},{ }^{3} J(\mathrm{H}, \mathrm{H})=8.5 \mathrm{~Hz}, 1 \mathrm{H}, \mathrm{DNP}\right.$ aryl $-\mathrm{H} m-\mathrm{O}), 7.29\left(\mathrm{t},{ }^{3} J(\mathrm{H}, \mathrm{H})=8.5 \mathrm{~Hz}, 1 \mathrm{H}, \mathrm{DNP}\right.$ aryl $\left.-\mathrm{H} m-\mathrm{O}\right), 6.78\left(\mathrm{~d},{ }^{3} J(\mathrm{H}, \mathrm{H})=7.6\right.$ 
$\mathrm{Hz}, 1 \mathrm{H}, \mathrm{DNP}$ aryl $-\mathrm{H} o-\mathrm{O}), 6.77\left(\mathrm{~d},{ }^{3} J(\mathrm{H}, \mathrm{H})=7.6 \mathrm{~Hz}, 1 \mathrm{H}, \mathrm{DNP}\right.$ aryl $\left.-\mathrm{H} o-\mathrm{O}\right), 4.23-4.20$ (m, 4H, DNP - $\left.\mathrm{OCH}_{2}\right), 3.93-3.90(\mathrm{~m}, 4 \mathrm{H}), 3.74-3.71(\mathrm{~m}, 4 \mathrm{H}), 3.66-3.59(\mathrm{~m}, 8 \mathrm{H}), 3.54(\mathrm{t}$, $\left.{ }^{3} J(\mathrm{H}, \mathrm{H})=4.6 \mathrm{~Hz}, 2 \mathrm{H}\right), 3.29\left(\mathrm{t},{ }^{3} J(\mathrm{H}, \mathrm{H})=4.9 \mathrm{~Hz}, 2 \mathrm{H}\right),{ }^{13} \mathrm{C} \mathrm{NMR}\left(125 \mathrm{MHz}, \mathrm{CDCl}_{3}\right): \delta$ 154.18, 154.13, 126.61, 126.59, 124.99, 124.93, 114.49, 114.41, 105.52 (2C), 72.43, 70.85, 70.81, 70.57, 70.29, 69.91, 69.70, 69.62, 67.74, 67.70, 61.52, 50.50. HRMS Calcd for $\mathrm{C}_{28} \mathrm{H}_{36} \mathrm{O}_{8}:$ 500.2410. HRMS Calcd for $\mathrm{C}_{22} \mathrm{H}_{31} \mathrm{~N}_{3} \mathrm{O}_{7}$ : 449.2162. Found: 449.2171.

Scheme S5. Synthesis of DNP Azidoalkyne Derivative 5.

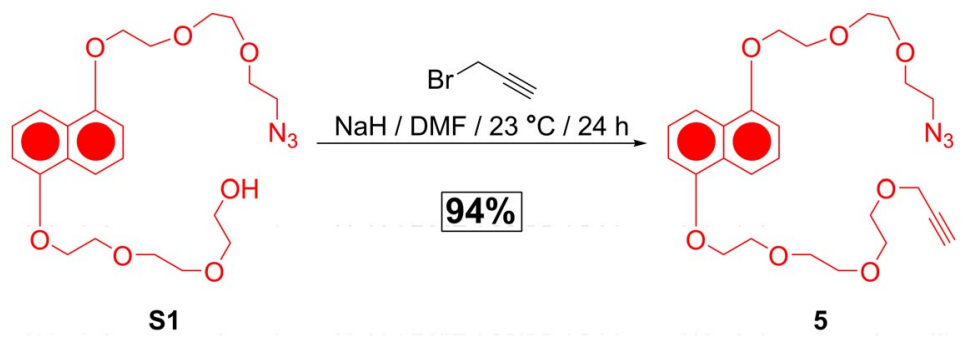

\section{Preparation of 1-(2-(2-(2-azidoethoxy)ethoxy)ethoxy)-5-(2-(2-(2-(prop-2-ynyloxy)- ethoxy)ethoxy)ethoxy)naphthalene 5:}

Solid $\mathrm{NaH}$ (5.0 mg, $0.208 \mathrm{mmol}$ ) was added to the solution of $\mathbf{S 1}$ (45.0 $\mathrm{mg}, 0.100 \mathrm{mmol}$ ) in dry DMF $(20 \mathrm{~mL})$. The resulting suspension was stirred at $23{ }^{\circ} \mathrm{C}$ until the evolution of gas ceased (approx. $25 \mathrm{~min}$ ). A solution of propargyl bromide in xylene ( $80 \%$ by weight, $56 \mu \mathrm{L}, 0.5 \mathrm{mmol}$ ) was injected using a syringe. The mixture was stirred at $23{ }^{\circ} \mathrm{C}$ for $24 \mathrm{~h}$, during which time it gradually turned brown. The reaction was stopped by the addition of $\mathrm{MeOH}$, and the solvents were removed in vacuo. Crude brown oil was first filtered through a plug of silica, eluting with $\mathrm{Me}_{2} \mathrm{CO}$, and then subjected to chromatography on silica (hexane/EtOAc $=50 / 50)$ to provide 5 as a brown oil $(46.0 \mathrm{~g}, 94 \%) .5: \mathrm{MS}(\mathrm{FAB}+): \mathrm{m} / \mathrm{z}(\mathrm{rel}$ intensity $)=526\left([\mathrm{M}+\mathrm{K}]^{+}, 25 \%\right), 487\left(\mathrm{M}^{+}, 100\right), 460\left(\left[\mathrm{M}-\mathrm{CH}_{2} \mathrm{C} \equiv \mathrm{CH}\right]^{+}, 27\right), 288(17) .{ }^{1} \mathrm{H}$ $\left.\operatorname{NMR}\left(500 \mathrm{MHz}^{\mathrm{CDCl}}\right)_{3}\right): \delta 7.84\left(\mathrm{~d},{ }^{3} J(\mathrm{H}, \mathrm{H})=8.2 \mathrm{~Hz}, 2 \mathrm{H}, \mathrm{DNP}\right.$ aryl $\left.-\mathrm{H} p-\mathrm{O}\right), 7.31\left(\mathrm{t},{ }^{3} J\right.$ $(\mathrm{H}, \mathrm{H})=8.2 \mathrm{~Hz}, 2 \mathrm{H}, \mathrm{DNP}$ aryl $-\mathrm{H} m-\mathrm{O}), 6.79\left(\mathrm{~d},{ }^{3} J(\mathrm{H}, \mathrm{H})=7.6 \mathrm{~Hz}, 2 \mathrm{H}, \mathrm{DNP}\right.$ aryl $-\mathrm{H} o-$ O), $4.22\left(\right.$ br t, $\left.{ }^{3} J(\mathrm{H}, \mathrm{H})=4.9 \mathrm{~Hz}, 4 \mathrm{H}, \mathrm{DNP}-\mathrm{OCH}_{2}\right), 4.14\left(\mathrm{~d},{ }^{4} J(\mathrm{H}, \mathrm{H})=2.4 \mathrm{~Hz}, 2 \mathrm{H}\right.$, propargyl $\left.\mathrm{CH}_{2}\right), 3.92\left(\right.$ br t, $\left.{ }^{3} J(\mathrm{H}, \mathrm{H})=4.6 \mathrm{~Hz}, 4 \mathrm{H}\right), 3.75-3.73(\mathrm{~m}, 4 \mathrm{H}), 3.65-3.59(\mathrm{~m}, 8 \mathrm{H})$, $3.29\left(\mathrm{br} \mathrm{t},{ }^{3} J(\mathrm{H}, \mathrm{H})=4.9 \mathrm{~Hz}, 4 \mathrm{H}\right), 2.43\left(\mathrm{t},{ }^{4} J(\mathrm{H}, \mathrm{H})=2.4 \mathrm{~Hz}, 1 \mathrm{H}, \equiv \mathrm{C}-H\right) .{ }^{13} \mathrm{C}$ NMR $(125$ 
$\mathrm{MHz}, \mathrm{CDCl}_{3}$ ): $\delta$ 154.21, 154.19, 126.62 (2C), 124.98 (2C), 114.50, 114.44, 105.53 (2C), $79.60(C \equiv \mathrm{C}-\mathrm{H}), 74.55(\mathrm{C} \equiv C-\mathrm{H}), 70.85,70.80,70.57,70.54,70.30,69.91,69.71,69.64$, 68.96, 67.75 (2C), 58.22, 50.51. HRMS Calcd for $\mathrm{C}_{25} \mathrm{H}_{33} \mathrm{~N}_{3} \mathrm{O}_{7}$ : 487.2318. Found: 487.2324 .

Scheme S6. Synthesis of Catenane $6 \cdot 4 \mathrm{PF}_{6}$.

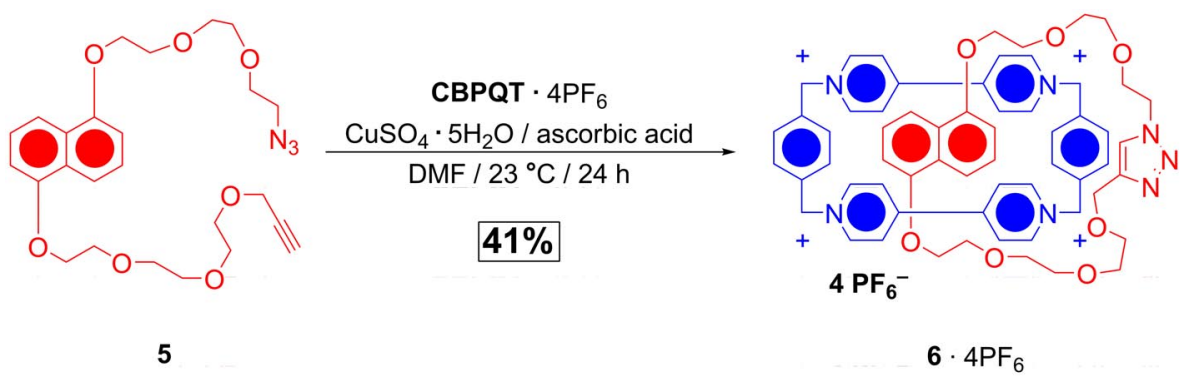

\section{Preparation of the $[2]$ Catenane $6 \cdot 4 \mathrm{PF}_{6}$ :}

The DNP derivative 5 (24.0 mg, $0.05 \mathrm{mmol})$ and CBPQT · 4PF 6 (55.0 mg, $0.05 \mathrm{mmol})$, were dissolved in $50 \mathrm{~mL}$ of DMF. Stock solutions of ascorbic acid (containing $0.88 \mathrm{mg}$, $0.005 \mathrm{mmol}$ ) and $\mathrm{CuSO}_{4} \cdot 5 \mathrm{H}_{2} \mathrm{O}$ (containing $1.25 \mathrm{mg}, 0.005 \mathrm{mmol}$ ) in DMF were diluted to $50 \mathrm{~mL}$. Into this mixture, the solution of 5 and CBPQT $\cdot 4 \mathrm{PF}_{6}$ was dripped in over $5 \mathrm{~h}$. After the addition was complete, the mixture was left to stir at $23{ }^{\circ} \mathrm{C}$ for 24 additional $\mathrm{h}$. The solvent was removed in vacuo, and the crude purple solid was purified by column chromatography on silica (gradient elution: $1 \% \rightarrow 5 \%$ solution of $\mathrm{NH}_{4} \mathrm{PF}_{6}$ in $\mathrm{Me}_{2} \mathrm{CO}$ ). Crude catenane $6 \cdot 4 \mathrm{PF}_{6}$ was precipitated by the addition of water and filtered. This procedure was repeated twice, to yield a purple powder, containing $6 \cdot 4 \mathrm{PF}_{6}$, contaminated with CBPQT - $4 \mathrm{PF}_{6}$ as minor fraction. This powder was washed with three portions of cold $\mathrm{MeOH}$. Evaporation of the solvent from the combined wash solutions yielded pure $\mathbf{6}$. $4 \mathrm{PF}_{6}$ as a purple solid, $\mathrm{mp} 190{ }^{\circ} \mathrm{C}$ with decomp. Yield: $32.0 \mathrm{mg}(41 \%) . \quad 6 \cdot 4 \mathrm{PF}_{6}: \mathrm{MS}$ $(\mathrm{FAB}+): \mathrm{m} / z($ rel intensity $)=1442\left(\left[\mathbf{6} \cdot 3 \mathrm{PF}_{6}\right], 50 \%\right), 1297\left(\left[\mathbf{6} \cdot 2 \mathrm{PF}_{6}\right], 100\right), 1152([6 \cdot$ $\mathrm{PF}_{6}$ ] , 36), 955 ([CBPQT $\left.\left.\cdot 3 \mathrm{PF}_{6}\right], 20\right), 810$ ([CBPQT $\left.\left.\cdot 2 \mathrm{PF}_{6}\right], 92\right), 665$ ([CBPQT $\cdot \mathrm{PF}_{6}$ ], 67). ${ }^{1} \mathrm{H}$ NMR $\left(500 \mathrm{MHz}, \mathrm{CD}_{3} \mathrm{CN}\right): \delta 9.15\left(\mathrm{~d},{ }^{3} J(\mathrm{H}, \mathrm{H})=6.4 \mathrm{~Hz}, 1 \mathrm{H}, \alpha-\mathrm{CBPQT}^{4+}-\mathrm{H}\right), 8.86$ (br s, $\left.1 \mathrm{H}, \alpha-\mathrm{CBPQT}^{4+}-\mathrm{H}\right), 8.83\left(\mathrm{~d},{ }^{3} J(\mathrm{H}, \mathrm{H})=7.0 \mathrm{~Hz}, 1 \mathrm{H}, \alpha-\mathrm{CBPQT}^{4+}-\mathrm{H}\right), 8.73\left(\mathrm{~d},{ }^{3} J\right.$ $\left.(\mathrm{H}, \mathrm{H})=6.4 \mathrm{~Hz}, 2 \mathrm{H}, \alpha-\mathrm{CBPQT}^{4+}-\mathrm{H}\right), 8.68\left(\mathrm{~d},{ }^{3} J(\mathrm{H}, \mathrm{H})=6.4 \mathrm{~Hz}, 1 \mathrm{H}, \alpha-\mathrm{CBPQT}^{4+}-\mathrm{H}\right)$, 
$8.65\left(\mathrm{~d},{ }^{3} J(\mathrm{H}, \mathrm{H})=6.4 \mathrm{~Hz}, 1 \mathrm{H}, \alpha-\mathrm{CBPQT}^{4+}-\mathrm{H}\right), 8.51\left(\mathrm{~d},{ }^{3} J(\mathrm{H}, \mathrm{H})=6.4 \mathrm{~Hz}, 1 \mathrm{H}, \alpha-\right.$ $\left.\mathrm{CBPQT}^{4+}-\mathrm{H}\right), 8.17\left(\mathrm{~d},{ }^{3} J(\mathrm{H}, \mathrm{H})=6.1 \mathrm{~Hz}, 1 \mathrm{H}, \beta-\mathrm{CBPQT}^{4+}-\mathrm{H}\right), 7.99\left(\mathrm{~d},{ }^{3} J(\mathrm{H}, \mathrm{H})=6.1\right.$ $\left.\mathrm{Hz}, 1 \mathrm{H}, \beta-\mathrm{CBPQT}^{4+}-\mathrm{H}\right), 7.97-7.92\left(\mathrm{~m}, 6 \mathrm{H}, \beta-\mathrm{CBPQT}^{4+}-\mathrm{H}\right), 7.53($ br s, $1 \mathrm{H}$, aryl$\left.\mathrm{CBPQT}^{4+}-\mathrm{H}\right), 7.40\left(\mathrm{br} \mathrm{d},{ }^{3} \mathrm{~J}(\mathrm{H}, \mathrm{H})=6.1 \mathrm{~Hz}, 1 \mathrm{H}\right.$, aryl-CBPQT $\left.{ }^{4+}-\mathrm{H}\right), 7.37-7.17(\mathrm{~m}, 5 \mathrm{H}$, aryl-CBPQT $\left.{ }^{4+}-\mathrm{H}\right), 7.05(\mathrm{~s}, 1 \mathrm{H}$, triazole $-\mathrm{H}), 6.86\left(\mathrm{br} \mathrm{d},{ }^{3} \mathrm{~J}(\mathrm{H}, \mathrm{H})=6.1 \mathrm{~Hz}, 1 \mathrm{H}\right.$, aryl$\left.\mathrm{CBPQT}^{4+}-\mathrm{H}\right), 6.33\left(\mathrm{~d},{ }^{3} J(\mathrm{H}, \mathrm{H})=7.9 \mathrm{~Hz}, 1 \mathrm{H}, \mathrm{DNP}\right.$ aryl $\left.-\mathrm{H} o-\mathrm{O}\right), 6.31\left(\mathrm{~d},{ }^{3} J(\mathrm{H}, \mathrm{H})=7.4\right.$ $\mathrm{Hz}, 1 \mathrm{H}, \mathrm{DNP}$ aryl $-\mathrm{H} o-\mathrm{O}), 5.97\left(\mathrm{t},{ }^{3} J(\mathrm{H}, \mathrm{H})=7.6 \mathrm{~Hz}, 1 \mathrm{H}, \mathrm{DNP}\right.$ aryl $\left.-\mathrm{H} m-\mathrm{O}\right), 5.86\left(\mathrm{t},{ }^{3} J\right.$ $(\mathrm{H}, \mathrm{H})=7.6 \mathrm{~Hz}, 1 \mathrm{H}, \mathrm{DNP}$ aryl $-\mathrm{H} m-\mathrm{O}), 5.74-5.55\left(\mathrm{~m}, 8 \mathrm{H}, \mathrm{CBPQT}^{4+}\right.$ benzyl $\left.\mathrm{H}\right), 4.56(\mathrm{~m}$, 2H), 4.33-3.51 (m, 24H), $2.32\left(\mathrm{~d},{ }^{3} J(\mathrm{H}, \mathrm{H})=7.9 \mathrm{~Hz}, 2 \mathrm{H}\right.$, DNP aryl $\left.-\mathrm{H} p-\mathrm{O}\right)$. HRMS Calcd for $\mathrm{C}_{61} \mathrm{H}_{65} \mathrm{~N}_{7} \mathrm{O}_{7} \mathrm{P}_{2} \mathrm{~F}_{12}\left(\left[\mathbf{6} \cdot 2 \mathrm{PF}_{6}\right]\right): 1297.423$. Found: 1297.426 .

Characterization of the [2]Catenanes $3 \cdot 4 \mathrm{PF}_{6}$ and $6 \cdot 4 \mathrm{PF}_{6}$ by ${ }^{1} \mathrm{H}$ NMR Spectroscopy:

The ${ }^{1} \mathrm{H}$ NMR spectra (recorded in $\mathrm{CD}_{3} \mathrm{CN}$ at $25{ }^{\circ} \mathrm{C}$ ) of $\mathbf{3} \cdot 4 \mathrm{PF}_{6}$ and $\mathbf{6} \cdot 4 \mathrm{PF}_{6}$ are shown in Figure S1 with partial assignments.

\section{References:}

(1) Asakawa, M.; Dehaen, W.; L’abbé, G.; Menzer, S.; Nouwen, J.; Raymo, F. M.; Stoddart, J. F.; Williams, D. J. J. Org. Chem. 1996, 61, 9591-9595.

(2) Ashton, P. R.; Huff, J.; Menzer, S.; Parsons, I. W.; Preece, J. A.; Stoddart, J. F.; Tolley, M. S.; White, A. J. P.; Williams, D. J. Chem. Eur. J. 1996, 2, 31-44.

(3) Anelli, P. L.; Ashton, P. R.; Ballardini, R.; Balzani, V.; Delgado, M.; Gandolfi, M. T.; Goodnow, T. T.; Kaifer, A. E.; Philp, D.; Pietraszkiewicz, M.; Prodi, L.; Reddington, M. V.; Slawin, A. M. Z.; Spencer, N.; Stoddart, J. F.; Vicent, C.; Williams, D. J. J. Am. Chem. Soc. 1992, 114, 193-218.

(4) Liu, Y.; Saha, S.; Vignon, S. A.; Flood, A. H.; Stoddart, J. F. Synthesis 2005, 34373455 . 
Figure S1. ${ }^{1} \mathrm{H}$ NMR Spectra of [2]Catenanes $3 \cdot 4 \mathrm{PF}_{6}$ and $\mathbf{6} \cdot 4 \mathrm{PF}_{6}$.

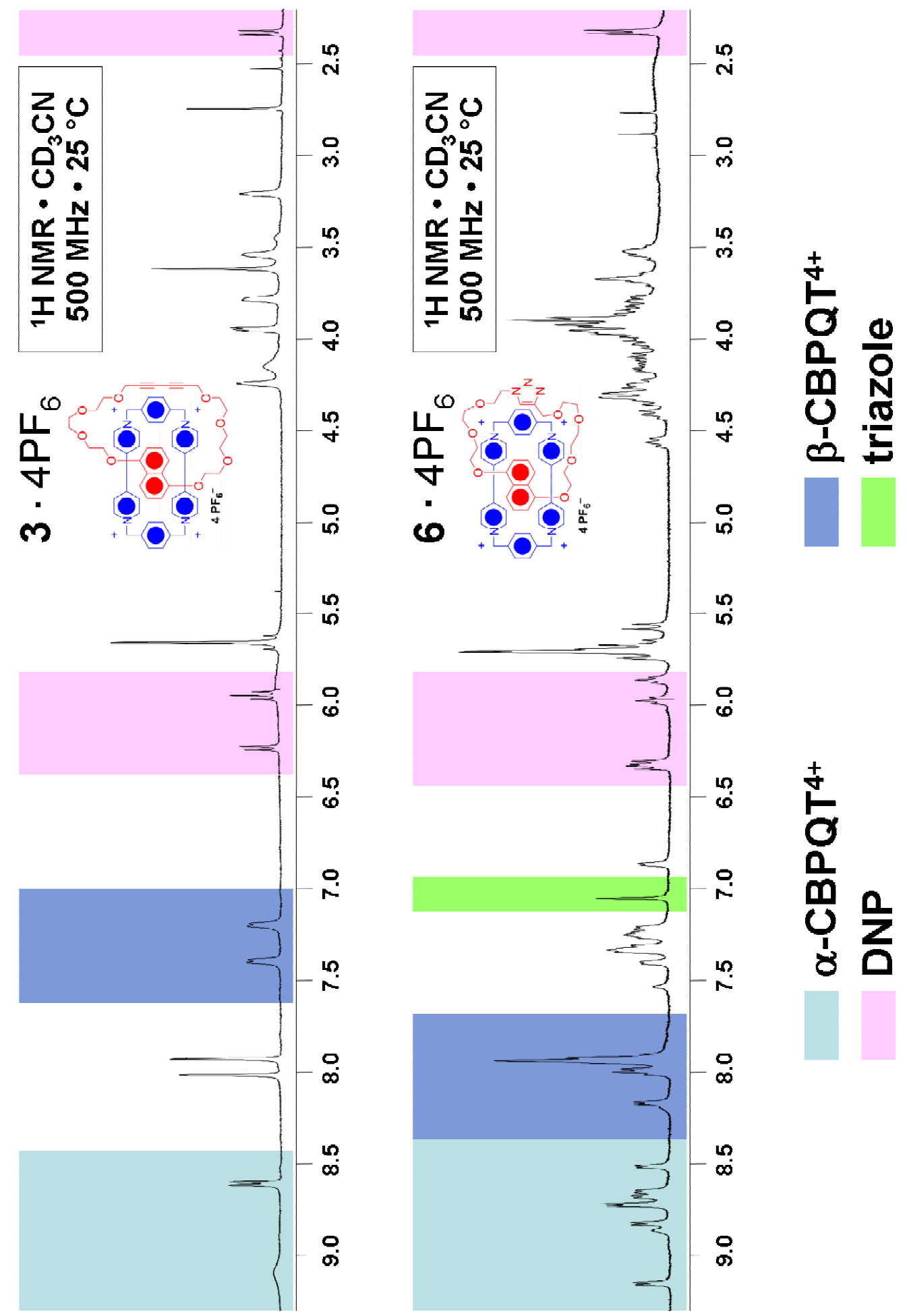

